\title{
RELAÇÃO ENTRE ESTADO COGNITIVO \\ E VARIÁVEIS SOCIODEMOGRÁFICAS \\ E FUNCIONAIS EM IDOSOS LONGEVOS: \\ ESTUDO OBSERVACIONAL NO MUNICIPIO \\ DE PORTO ALEGRE/RS
}

\author{
Tainara Steffens \\ Talita Molinari2 \\ Caroline Pietta Dias ${ }^{3}$
}

resumo

Introdução: com o aumento da expectativa de vida, o número de idosos longevos vem aumentando. $\mathrm{O}$ envelhecimento é considerado um processo natural que afeta a maioria dos organismos vivos, e provoca perdas progressivas das funções. Dentre estas funções podemos destacar algumas alterações de ordem mental e neurológica, como também redução da capacidade funcional e autonomia nas atividades de vida diária. Assim, o objetivo deste estudo foi verificar a associação

1 Graduada em Bacharel em Fisioterapia. Mestranda no Programa de Pós-Graduação em Ciência do Movimento Humano na Universidade Federal do Rio Grande do Sul (UFRGS). E-mail: tainarast@hotmail.com. 2 Graduada em Bacharel em Fisioterapia. Mestranda no Programa de Pós-Graduação em Ciência do Movimento Humano na Universidade Federal do Rio Grande do Sul (UFRGS). E-mail: t.molinari@hotmail.com. 3 Graduada em Educação Física. Doutora em Ciências do Movimento Humano. Docente no curso de Graduação e Pós-Graduação da Escola de Educação Física, Fisioterapia e Dança da Universidade Federal do Rio Grande do Sul (UFRGS). E-mail: carolpieta@yahoo.com.br. 
entre o estado cognitivo, variáveis sociodemográficas e o nível de dependência funcional em idosos longevos. Materiais e métodos: os idosos foram selecionados por acessibilidade em Estratégias Saúde da Família, Instituições de Longa Permanência e anúncios públicos. Foram realizadas entrevistas domiciliares e aplicados os seguintes instrumentos: 1) Avaliação sociodemográfica; 2) Mini Exame do Estado Mental; 3) Índice de Katz; e 4) Escala de Lawton e Brody. Resultados: foram avaliados 66 idosos com idade média de 94,89 4 4,16 anos, na sua maioria do sexo feminino, residentes em domicílios, com escolaridade de 1 a 4 anos e aposentados. Para as avaliações, a maioria dos idosos foram classificados sem declínio cognitivo, apresentando independência nas atividades básicas da vida diária e independência parcial nas atividades instrumentais da vida diária. $\bigcirc$ estado cognitivo apresentou uma correlação fraca e positiva com o local de moradia dos idosos ( $r=0,314, p<0,01$ ) e correlação positiva e moderada com as atividades básicas ( $r=0,450, p<0,01)$ e instrumentais da vida diária ( $r=0,433, p<0,01$ ). Conclusão: o estado cognitivo apresentou associação significativa com o local de moradia e com as atividades básicas e instrumentais da vida diária, sugerindo que morar no domicílio e possuir boa independência funcional associam-se a um menor declínio cognitivo.

palavras - chave

Saúde Mental. Estado Funcional. Nonagenários. Centenários.

Na população Brasileira, dentre os mais de 206 milhões de habitantes, quase $1,7 \%$ são idosos longevos, ou seja, idosos com idade igual ou superior a 80 anos (MAGNOLFI et al., 2007; GRDEN et al., 2017; CHAVES et al., 2017). O processo de envelhecimento teve crescimento em várias regiões do país, principalmente no período de 1970 a 2010 (CLOSS; SCHWANKE, 2012) e projeções para o ano de 2030 indicam um aumento de quase 3\% de longevos em todo o país (IBGE, 2010).

O envelhecimento é considerado um processo natural que afeta a maioria dos organismos vivos, e provoca perdas progressivas das funções (SANTOS; ANDRADE; BUENO, 2009; LÓPEZ-OTÍN et al., 2013). Este processo pode estar acompanhado de algumas alterações mentais e neurológicas, tais como as alterações das funções cognitivas, memória, atenção, linguagem, praxia, habilidade 
visuoconstrutiva e funções executivas (FICHMAN et al., 2013). Vários fatores podem estar relacionados ao comprometimento cognitivo nos idosos, entre eles, a qualidade de vida (CAMPOS; FERREIRA; VARGAS, 2015). A mesma tem relação direta com fatores complexos que interferem na saúde física, estado psicológico, convivências sociais e nível de independência do indivíduo (OMS, 2015), que resultam em declínio das funções cognitivas, que interferem na autonomia e saúde física do idoso (CAMPOS; FERREIRA; VARGAS, 2015; SOBRAL, A.; ARAUJO; SOBRAL, M., 2018). Além do declínio cognitivo, uma das principais alterações que os idosos vivenciam é a perda da capacidade funcional, a qual é entendida como a habilidade da pessoa efetuar determinada ação ou tarefa de modo independente (FUHRMANN et al., 2015). A redução da capacidade funcional do idoso está relacionada com o próprio processo de envelhecimento e surgimento de doenças crônico degenerativas que afetam a funcionalidade, com consequente dependência funcional (REIS; MOREIRA; DUARTE, 2015) caracterizada por restrição ou ajuda na participação das atividades do dia a dia (REIS; MOREIRA; DUARTE, 2015).

Sendo assim, o declínio das funções cognitivas e motoras repercute tanto nas Atividades Básicas da Vida Diária (ABVD) como nas Atividades Instrumentais da Vida Diária (AIVD) (FLECK et al., 2014; LOPES; SANTOS, 2015). Entre as habilidades que compõem a funcionalidade, nas ABVD, estão os comportamentos básicos e habituais de autocuidado, como a capacidade de alimentar-se, banhar-se e vestir-se, enquanto nas AIVD, tarefas mais complexas e relacionadas à autonomia e participação social, como capacidade de realizar compras, atender ao telefone, tomar medicação, gerenciamento financeiro e utilizar meios de transporte (FARÍAS-ANTÚNEZ et al., 2018).

O declínio da capacidade cognitiva dos idosos implica em maiores cuidados por parte dos familiares, e pode estar associado a maior dependência do idoso tanto nas ABVD como nas AIVD, podendo impactar negativamente na qualidade de vida e muitas vezes ocasionando a institucionalização desses idosos (IMAGINÁRIO et al., 2017) e até mesmo de morte prematura (FARÍAS-ANTÚNEZ et al., 2018). Diante do exposto, o objetivo deste estudo foi verificar a associação entre o estado cognitivo, variáveis sociodemográficas e o nível de dependência funcional nas ABVD e AIVD em idosos longevos acima de 90 anos de idade.

\section{Métodos}

O presente estudo caracteriza-se como sendo analítico, observacional e transversal (HULLEY et al., 2015) com amostragem não probabilística por acessibilidade (RICHARDSON et al., 2012; GIL, 2008). A população do estudo 
foi composta por idosos longevos com idade acima de 90 anos residentes no município de Porto Alegre, no estado do Rio Grande do Sul, Brasil.

Os idosos foram recrutados por meio da Atenção Primária com direcionamento para uma gerência distrital que abrange três Unidades Básicas de Saúde, onde se concentra maior parte dos idosos e que apresenta Estratégia Saúde da Família (ESF) em Porto Alegre/RS. Também foram contatadas associações de aposentados e Instituições de Longa Permanência para Idosos (ILPI) e divulgação via anúncio público. Por meio de levantamento de dados fornecidos pela atenção primária e por associações de aposentados o total foi de 201 idosos longevos, sendo que destes apenas 23 idosos participaram do estudo. Posteriormente, foram incluídos mais 43 idosos após contato com as ILPI e por voluntários através de anúncio público, chegando ao total de 66 idosos que constituíram a amostra do estudo (Figura 1).

O projeto de pesquisa foi aprovado pelo Comitê de Ética em Pesquisa da Universidade Federal do Rio Grande do Sul (CAAE: 79748517.5.0000.5347), e pelo Comitê de Ética em Pesquisa da Secretaria Municipal (CAAE: 79748517.5.3001.5338) e Estadual (CAAE: 79748517.5.3002.5312) de Saúde de Porto Alegre. Todos os idosos assinaram um termo de consentimento livre e esclarecido concordando com a participação no estudo.

Figura 1 - Fluxograma da seleção da amostra.

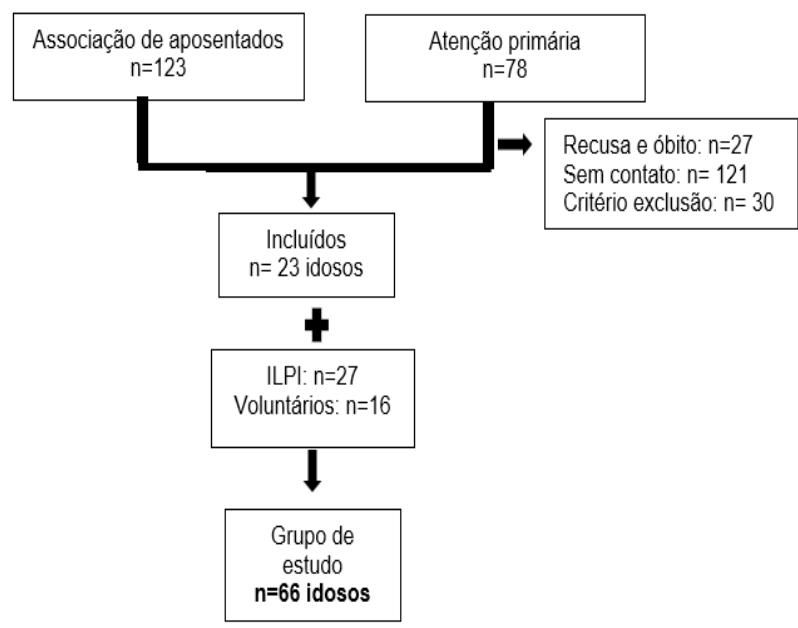

Legenda: ILPI = Instituições de Longa Permanência para Idosos.

Fonte: Elaborada pelas autoras, 2018. 
Os idosos foram submetidos aos seguintes protocolos de avaliação:

1. Questionário referente a informações sociodemográficas (gênero, idade, estado civil, escolaridade, número de medicações de uso contínuo);

2. Mini Exame do Estado Mental (MEEM) para avaliar a capacidade cognitiva;

Trata-se de um questionário desenvolvido por M. Folstein, S. Folstein e Mchugh (1975) e validado para a população brasileira por Bertolucci et al. (1994). É composto por 11 questões abertas agrupadas em 6 categorias: orientação temporal e espacial; processamento; atenção; cálculo; evocação; linguagem e habilidade construtiva. Dentre as versões existentes na literatura, optamos pela versão utilizada por Lourenço e Veras (2006), bem como os pontos de corte com valores mínimos de 23 pontos para idosos com 5 anos ou mais de escolaridade, e de 19 pontos para aqueles com escolaridade igual ou inferior a 4 anos. Pontuação inferior aos pontos de corte indica declínio cognitivo (LEITE et al., 2015). A pontuação total do questionário é de 30 pontos onde os valores mais altos indicam indivíduos sem declínio cognitivo.

3. Índice de Katz para avaliação da independência funcional nas Atividades Básicas da Vida diária (ABVD) (ex. banho, vestuário, higiene, transferência, continência e alimentação);

O instrumento foi elaborado por Katz et al. (1963) e validado para o Brasil por Lino et al. (2008). É composto por 6 questões com 3 alternativas de respostas para a classificação da dependência de acordo com a pontuação: 0 = independente; 1 = quando em alguns casos necessita de ajuda; e 2 = dependente de ajuda. Os itens são classificados quanto à assistência, à qualidade da execução e a iniciativa do sujeito. Na pontuação final, o somatório das respostas gera a classificação funcional: entre 0-2 pontos independentes, 3-4 dependentes parcial, acima de 4 dependentes (MACIEL; GUERRA, 2008).

4. Escala de Lawton e Brody (1969) para avaliação das Atividades Instrumentais de Vida Diária (AIVD) (ex. uso do telefone, viagens, fazer compras, preparação de refeições, trabalho doméstico, responsabilidade com a medicação e o manejo do dinheiro).

A escala foi adaptada para a versão brasileira por Santos e Junior (2008) possuindo 3 alternativas de respostas que recebem a seguinte pontuação: 3 = independente; 2 = dependência parcial; e 1 = dependência total. Os itens são classificados quanto à assistência, à qualidade da execução e a iniciativa do sujeito. Na pontuação final, o somatório das respostas gera a classificação funcional: dependência total $\leq 5$, dependência parcial $>5<21$, e independência $=21$ (SANTOS; VIRTUOSO JÚNIOR, 2008). 
Todos os instrumentos foram aplicados em forma de entrevista, a fim de minimizar possíveis erros de interpretação das questões devido a particularidade da amostra, tais como presença de baixa acuidade visual, analfabetismo ou semianalfabetíssimo, e alterações na coordenação motora. A coleta de dados foi realizada na residência dos idosos e nas ILPI e ocorreu no período de maio a agosto de 2018.

Foi realizada estatística descritiva (média $\pm d p$, frequências absolutas e relativas) para todos os dados. A normalidade dos dados foi avaliada pelo teste Kolmogorov-Smirnov. A correlação de Spearman foi utilizada para investigar a relação entre o estado cognitivo com as características sociodemográficas (idade, anos de estudo e local de moradia) e o nível de independência funcional nas ABVD e AIVD. As correlações foram consideradas fracas $(\mathrm{r}<0,3)$, moderadas $(\mathrm{r} \geq 0,3$ e $<0,7)$ ou fortes $(r \geq 0,7)$ de acordo com Melo et al. (2017). Foi adotado um $\alpha=0,05$, e os dados foram analisados no programa SPSS versão 21.

\section{Resultados}

Foram avaliados 66 idosos longevos com média de idade de 94,89 $\pm 4,16$ anos, com predomínio do gênero feminino (77,3\%), e escolaridade entre 1 a 4 anos de estudo (51,5\%), residentes em domicílios $(56,1 \%)$, aposentados $(60,6 \%)$ e viúvos (59,1\%), de acordo com a Tabela 1.

Tabela 1 - Características sociodemográficas dos participantes.

\begin{tabular}{llll}
\hline & & $\mathrm{n}$ & $\%$ \\
\hline \multirow{3}{*}{ Idade } & $90-94$ anos & 37 & 56,1 \\
& $95-99$ anos & 18 & 27,3 \\
& 100 anos ou mais & 11 & 16,7 \\
\hline \multirow{2}{*}{ Gênero } & Feminino & 51 & 77,3 \\
& Masculino & 15 & 22,7 \\
\hline \multirow{2}{*}{ Anos de Estudo } & Analfabetos & 4 & 6,1 \\
& 5 anos ou mais & 34 & 51,5 \\
\hline \multirow{2}{*}{ Local de } & Casa & 28 & 42,4 \\
\hline
\end{tabular}




\begin{tabular}{llcc}
\hline & Solteiro & 16 & 24,2 \\
\multirow{3}{*}{ Estado Civil } & Casado & 10 & 15,2 \\
& Viúvo & 39 & 59,1 \\
& Separado/Divorciado & 1 & 1,5 \\
\hline \multirow{3}{*}{ Fonte Renda } & Aposentado & 40 & 60,6 \\
& Pensionista & 12 & 18,2 \\
& Aposentado e Pensionista & 13 & 19,7 \\
& Nenhum & 1 & 1,5 \\
\hline
\end{tabular}

Fonte: Elaborada pelas autoras, 2018.

A maioria dos idosos (66,7\%) apresentaram independência nas ABVD e 89,4\% apresentaram dependência parcial nas AIVD; 71,2\% dos idosos foram classificados sem declínio cognitivo.

Tabela 2 - Estado cognitivo, nível de dependência funcional nas ABVD e nas AIVD.

\begin{tabular}{llcc}
\hline & Classificação & $\mathrm{n}$ & $\%$ \\
\hline ABVD & Independente & 44 & 66,7 \\
& Dependente Parcial & 17 & 25,8 \\
& Dependente Total & 5 & 7,6 \\
\hline \multirow{2}{*}{ AIVD } & Independente & 2 & 3 \\
& Dependente Parcial & 59 & 89,4 \\
& Dependente Total & 5 & 7,6 \\
\hline Estado cognitivo & Sem Declínio & 47 & 71,2 \\
& Com Declínio & 19 & 28,8 \\
\hline
\end{tabular}

Legenda: $A B V D$ = Atividades Básicas da Vida Diária; AIVD = Atividades Instrumentais da Vida Diária. Fonte: Elaborada pelas autoras, 2018.

O estado cognitivo apresentou uma correlação fraca e positiva com o local de moradia dos idosos $(r=0,314, \mathrm{p}<0,01)$, indicando que residir em domicílio está associado com o melhor estado cognitivo. Da mesma forma, com a ABVD apresentou correlação positiva e moderada $(r=0,450, p<0,01)$ e $\operatorname{AIVD}(\mathrm{r}=0,433, \mathrm{p}<0,01)$, o que demostra que um bom estado cognitivo está associado a independência funcional nas ABVD, e dependência parcial nas AIVD (Tabela 3). O estado cognitivo não apresentou associação significativa com as características sociodemográficas investigadas (idade, escolaridade, estado civil e fonte de renda), de acordo com a Tabela 3. 
Tabela 3 - Correlações Spearman entre Estado cognitivo e as características sociodemográficas, ABVD e AlVD.

\begin{tabular}{lcc}
\hline & \multicolumn{2}{c}{ Estado Cognitivo } \\
\hline Idade & $r$ & $p$ \\
Anos de Estudo & 0,144 & $>0,01$ \\
Local de Moradia & 0,026 & $>0,01$ \\
Estado Civil & 0,314 & $<0,01$ \\
Fonte de Renda & $-0,050$ & $>0,01$ \\
ABVD & $-0,133$ & $>0,01$ \\
AlVD & 0,450 & $<0,01$ \\
\hline
\end{tabular}

Fonte: Elaborada pelas autoras, 2018.

\section{Discussão}

O objetivo do estudo foi verificar a associação entre o estado cognitivo, e as variáveis sociodemográficas, o nível de dependência funcional nas ABVD e AIVD em idosos longevos acima de 90 anos, pertencentes da região urbana de Porto Alegre.

Os resultados mostraram que a maioria dos idosos (71,2\%) não apresentaram comprometimentos cognitivos, sendo que $66 \%$ apresentaram independência nas ABVD e $89 \%$ dependência parcial nas AIVD. Resultados diferentes foram observados em idosos com idade superior a 60 anos, onde 43,8\% estavam na faixa de 60 a 70 anos de idade, em uma área urbana do Rio Grande do Sul, e quase todos $(93,8 \%)$ apresentaram independência, tanto para a realização das ABVD como para as AIVD (LEITE et al., 2015). Desta forma, tem sido sugerido que $o$ avançar da idade pode ser um fator que interfira na dependência funcional dos idosos (FARÍAS-ANTÚNEZ et al., 2018). Em um estudo realizado na região rural de Pelotas/RS também observou que os idosos mais jovens, ou seja, aqueles com idade entre 60-69 anos em sua maioria foram classificados como independentes nas ABVD (57,9\%), já a prevalência de independência entre os idosos com 90 anos ou mais foi de apenas $0,4 \%$. A faixa etária que compreendeu a maior prevalência de incapacidade funcional foi a de 70-89 anos, totalizando $69,5 \%$ dos idosos dependentes (PINTO et al., 2016). Em outro estudo com idosos (idades entre 60 e 99 anos) foi observado que 90\% realizavam as ABVD e 83,7\% as AIVD de forma independente. De acordo com os autores, por pertencerem a 
um município pequeno, aonde a base econômica era a agricultura, esses idosos tinham histórico de vida ativo, inclusive nos deslocamentos diários que eram realizados a pé ou de bicicleta (BERLEZI et al., 2016).

Em um estudo que avaliou idosos da zona urbana de Pelotas/RS foi observado que o gênero feminino, com idades $\geq 80$ anos e que apresentaram duas ou mais morbidades tiveram maior prevalência de incapacidades nas ABVD, enquanto os idosos com escolaridade de 12 anos ou mais e os ativos no lazer tiveram menores prevalências de incapacidades (FARÍAS-ANTÚNEZ et al., 2018). Visto que, em nosso estudo o estado cognitivo apresentou correlação positiva e moderada com as ABVD $(r=0,450, p<0,01)$ e como a maioria dos idosos não apresentaram déficits cognitivos, podemos sugerir que as $\mathrm{ABVD}$ são tarefas mais simples e que dependem mais de condições físicas como mobilidade articular preservada e força muscular do que um estado cognitivo preservado.

Em nosso estudo, uma associação positiva moderada também foi observada entre o estado cognitivo e AIVD $(r=0,433, p<0,01)$, indicando que quanto melhor o estado cognitivo maior é a independência nas AIVD. Possivelmente, essa associação ocorreu pelo fato do desempenho cognitivo ser importante também para a realização das AIVD, uma vez que essas são tarefas de maior exigência cognitiva, como habilidades de memória e controle financeiro (PINTO et al., 2016). No que se refere às AIVD, no estudo de Farías-Antúnez et al. (2018) idosos com $>80$ anos, viúvos, com duas ou mais morbidades apresentaram maior prevalência de incapacidade. Talvez as AIVD não requerem apenas um bom estado cognitivo dos idosos já que envolve atividades mais complexas como compras, administração de medicações e finanças, o que exige o mínimo de escolaridade por parte dos mesmos para apresentarem independência nessas tarefas. Esse fato corrobora com os achados de Brito et al. (2014) em idosos longevos ( $86,1 \pm 6,39$ anos), que observou que os mesmos apresentaram dependência nas AIVD (94,6\%), sendo que só foram avaliados os que apresentaram bom estado cognitivo (BRITO et al., 2014). Além disso nas AIVD, a idade (60-69 anos), escolaridade (1-4 anos estudo), e a renda foram fatores relevantes e significativos entre os independentes funcionais (PINTO et al., 2016).

Sabe-se que o envelhecimento e todas suas consequências aumentam a vulnerabilidade e contribuem para as institucionalizações dos idosos (MACÊDO, VIEIRA, COSTA, 2018). No presente estudo, dos idosos avaliados apenas 43,9\% estavam em ILPI. Trindade et al. (2013) analisaram o declínio da cognição e o seu impacto nas habilidades funcionais em idosos institucionalizados e não institucionalizados, e observaram que os idosos institucionalizados em sua maioria tinham idade acima de 80 anos, e todos eram analfabetos, além de apresentarem baixos escores no MEEM. Já os não institucionalizados tinham 
idade entre 60 e 69 anos, todos tinham escolaridade e valores de MEEM de acordo com o ponto de corte. Neste sentido, nossos achados demonstraram que o estado cognitivo apresentou uma correlação fraca e positiva com o local de moradia dos idosos $(\mathrm{r}=0,314, \mathrm{p}<0,01)$, indicando que o bom estado cognitivo pode estar associado ao local de moradia, já que a maioria dos idosos residiam em ambiente domiciliar (56,1\%). Trindade et al. (2013) encontraram correlação forte e significativa $(r=0,717, p<0,01)$ entre o MEEM e habilidades funcionais nas atividades de vida diária para os idosos institucionalizados e moderada $(\mathrm{r}=0,463, \mathrm{p}<0,01)$ para aqueles não institucionalizados.

As variáveis sociodemográficas (idade, anos de estudo, estado civil e fonte de renda) não apresentaram associação significativa com o estado cognitivo dos idosos avaliados. Melo et al. (2017), observaram que apenas a idade foi considerada preditor de declínio cognitivo, e quando comparado com as outras variáveis tais como a instituição (ILPI, Hospital Universitário e Unidade saúde Escola), sexo e morbidades, as chances de prevalência de declínio cognitivo aumentam $8,7 \%$ para cada ano de vida a mais. Para os autores, esta redução cognitiva gradativa pode ser atribuída a senilidade sendo um fator que aumenta as chances para os déficits cognitivos.

Como limitações do estudo, podemos citar o número reduzido na amostra e o delineamento do estudo. A primeira limitação pode ser atribuída a dificuldade de conseguirmos uma aceitabilidade dos participantes em aceitarem realizar a avaliação em seus domicílios, por receio do atual cenário de insegurança. Já um delineamento de pesquisa observacional transversal, por prever apenas dados descritivos advindos de uma observação não permite a relação entre causa e efeito das variáveis.

\section{Conclusão}

Dos idosos longevos avaliados, a maioria não apresentou déficits cognitivos e eram independentes nas ABVD e dependentes nas AIVD. O estado cognitivo apresentou associação significativa com o local de moradia e com as ABVD e AIVD, sugerindo que morar no domicílio e possuir boa independência funcional associam-se a um menor declínio cognitivo.

Este estudo tem relevância social de cunho epidemiológico devido ao aumento da população longeva no Brasil e por escassez de estudos que avaliem exclusivamente a população acima de 90 anos de idade. Estas informações elucidam aspectos funcionais e cognitivos, que contribuirão para o planejamento de medidas preventivas que visem preservar a capacidade cognitiva e consequente independência funcional dos idosos longevos. 
RELATION BETWEEN COGNITIVE STATE

AND SOCIODEMOGRAPHIC

AND FUNCTIONAL VARIABLES IN LONG-LIVED

ELDERLY: OBSERVACIONAL STUDY

IN THE MUNICIPALITY OF PORTO ALEGRE/RS

abstract

Introduction: with increasing of life expectancy, the number of elderly people has been increasing. Aging is considered a natural process that causes progressive fuction loss. Among these functions we can highlight some mental and neurological changes, as well as reduction of functional capacity and autonomy in daily life activities. Thus, the aim of this study was to verify the association between the cognitive status, sociodemographic variables and the level of functional dependence in the long-lived people. Materials and methods: participants were selected for accessibility in Family Health Strategies, Long Stay Institutions and public announcements. The following instruments were used: 1) Socio-demographic evaluation; 2) Mini Mental State Examination; 3) Katz Index; and 4) Lawton and Brody Scale. Results: a total of 66 long-lived people with a mean age of $94.89 \pm 4.16$ years, mostly female, residing in households, with 1 to 4 years of schooling and retirees, were evaluated. The majority of the elderly were classified without cognitive decline, presenting independence in the daily life basic activities and partial independence in the daily life instrumental activities. The cognitive state presented a weak and positive correlation with the elderly residence place $(r=0,314, p<0,01)$ and a positive and moderate correlation with the daily life basic $(r=0.450, p<0.01)$ and instrumental activities $(r=0.433, p<0.01)$. Conclusion: the cognitive status showed a significant association with the residence place and with the daily life basic and instrumental activities, suggesting that living at home and have good functional independence are associated with a lower cognitive decline.

keywords

Mental Health. Functional Status. Nonagenarians. Centenarians. 
BERLEZI, Evelise et al. Como está a capacidade funcional de idosos residentes em comunidades com taxa de envelhecimento populacional acelerado? Revista Brasileira de Geriatria e Gerontologia, Rio de Janeiro, v. 19, n. 4, p. 643-652, 2016.

BERTOLUCCI, Paulo et al. Mini-Exame do Estado Mental em uma população geral: impacto da escolaridade. Arquivos de Neuro-Psiquiatria, São Paulo, v. 52, n. 1, p. 1-7, 1994.

BRITO, Thaís et al. Capacidade funcional e fatores associados em idosos longevos residentes em comunidade: estudo populacional no Nordeste do Brasil. Fisioterapia e Pesquisa, São Paulo, v. 21, n. 4, p. 308-313, 2014

CAMPOS, Ana; FERREIRA, Efigenia; VARGAS, Andréa. Determinantes do envelhecimento ativo segundo a qualidade de vida e gênero. Ciência e Saúde Coletiva, Rio de Janeiro, v. 20, n. 7, p. 2221-2237, 2015.

CHAVES, Renato et al. Perda cognitiva e dependência funcional em idosos longevos residentes em Instituições de Longa Permanência. Revista Cogitare Enfermagem, Curitiba, v. 22, n. 1, 2017

CLOSS, Vera; SCHWANKE, Carla. A evolução do índice de envelhecimento no Brasil, nas suas regiões e unidades federativas no período de 1970 a 2010. Revista Brasileira de Geriatria e Gerontologia, Rio de Janeiro, v. 15, n. 3, p. 443-458, 2012.

FARÍAS-ANTÚNEZ, Simone et al. Incapacidade funcional para atividades básicas e instrumentais da vida diária: um estudo de base populacional com idosos de Pelotas, Rio Grande do Sul, 2014. Epidemiologia Serviço de Saúde, Brasília, DF, v. 27, n. 2, 2018.

FICHMAN, Helenice et al. Predomínio de Comprometimento Cognitivo Leve Disexecutivo em idosos atendidos no ambulatório da geriatria de um hospital público terciário na cidade do Rio de Janeiro. Neuropsicologia Latinoamericana, [s.l.], v. 5, n. 2, p. 31-40, 2013

FLECK, Caren et al. Caracterização da capacidade funcional, nível cognitivo e força muscular respiratória de idosas com síndrome parkinsoniana. Estudos Interdisciplinares sobre o Envelhecimento, Porto Alegre, v. 19, n. 1, 2014.

FOLSTEIN, Marshal; FOLSTEIN, Susan; MCHUGH, Paul. "Mini-mental state": a practical method for grading the cognitive state of patients for the clinician. Journal of Psychiatric Research, Amsterdam, v. 12, n. 3, p. 189-198, 1975

FUHRMANN, Ana et al. Associação entre a capacidade funcional de idosos dependentes e a sobrecarga do cuidador familiar. Revista Gaúcha de Enfermagem, Porto Alegre, v. 36, n. 1, p. 14-20, 2015

GlL, Antônio. Métodos e técnicas de pesquisa social. 6. ed. São Paulo: Atlas, 2008. p. 97.

GRDEN, Clóris et al. Associação da síndrome da fragilidade física às características sociodemográficas de idosos longevos da comunidade. Revista Latino-Americana de Enfermagem, Ribeirão Preto, v. 25, 2017.

HULLEY, Stephen et al. Delineando a pesquisa clínica. 4. ed. Porto Alegre: Artmed, 2015. p. 90

IMAGINÁRIO, Cristina et al. Atividades de vida diária como preditores do estado cognitivo em idosos institucionalizados. Revista Portuguesa de Enfermagem de Saúde Mental, Porto, n. 18, p. 37-43, 2017.

INSTITUTO BRASILEIRO DE GEOGRAFIA E ESTATÍSTICA (IBGE). Sintese dos indicadores sociais: uma análise das condições de vida da população Brasileira, 2010. Rio de Janeiro: IBGE, 2010. Disponível em: . Acesso em: 11 ago. 2018. 
KATZ, Sidney et al. Studies of illness in the aged. The index of ADL: a standardized measure of biological ans psychosocial function. JAMA, Chicago, v. 185, n. 12 , p. 914-919, 1963

LAWTON, Powell; BRODY, Elaine. Assesment of older people: self-maintaining and instrumental activities of daily living. The Gerontologist, Washington, v. 9, n. 3, p. 179-186, 1969

LEITE, Marinês et al. Capacidade funcional e nível cognitivo de idosos residentes em uma comunidade do Sul do Brasil. Enfermagem Global, Múrcia, v. 14, n. 1, p. 1-11, 2015.

LINO, Valéria et al. Adaptação transcultural da Escala de Independência em Atividade da Vida Diária (Escala de Katz). Cadernos da Saúde Pública, Rio de Janeiro, v. 24, n. 1, p. 103-112, 2008.

LOPES, Geovanna; SANTOS, Maria. Funcionalidade de idosos cadastrados em uma unidade da Estratégia Saúde da Família segundo categorias da Classificação Internacional de Funcionalidade. Revista Brasileira de Geriatria e Gerontologia, Rio de Janeiro, v. 18, n. 1, p. 71-83, 2015.

LÓPEZ-OTíN, Carlos et al. The hallmarks of aging. Cell, Cambridge, v. 153, n. 6, p. 1194 $-1217,2013$

LOURENÇO, Roberto; VERAS, Renato. Mini-Exame do Estado Mental: características psicométricas em idosos ambulatoriais. Revista de Saúde Pública, São Paulo, v. 40, n. 4, p. 712-719, 2006

MACÊDO, Louise; VIEIRA, Gerlane; COSTA, Marta. Relação entre a capacidade funciona e a institucionalização da pessoa idosa: uma revisão integrativa. Revista de Pesquisa: Cuidado é Fundamental, Rio de Janeiro, v. 10, n. 2, p. 542-548, 2018

MACIEL, Álvaro; GUERRA, Ricardo. Limitação funcional e sobrevida em idosos de comunidade. Revista da Associação Médica Brasileira, São Paulo, v. 54, n. 4 p. 347-352, 2008.

MAGNOLFI, Stefano et al. Longevity index (LI\%) and centenarity index (Cl\%): new indicators to evaluate the characteristics of aging process in the Italian population. Archives of Gerontology and Geriatrics, Amsterdam, v. 44, n. 3, p. 271-276, 2007.

MELO, Beatriz et al. Avaliação cognitiva e funcional de idosos usuários do serviço público de saúde. Escola Anna Nery, Rio de Janeiro, v. 21, n. 4, 2017.

ORGANIZAÇÃO MUNDIAL DA SAÚDE (OMS). Resumo: Relatório mundial de Envelhecimento e Saúde. Genebra: OMS, 2015. Disponível em: http://sbgg.org.br/wp-content/ uploads/2015/10/OMS-ENVELHECIMENTO-2015-port.pdf. Acesso em: 11 ago. 2018.

PINTO, Andressa et al. Capacidade funcional para atividades da vida diária de idosos da Estratégia de Saúde da Família da zona rural. Ciência e Saúde Coletiva, Rio de Janeiro, v. 21, n. 11, p. 3545-3555, 2016.

REIS, Luciana; MOREIRA, Jaqueline; DUARTE, Stênio. Risco de queda, capacidade funcional e respiratória em idosos residentes em Instituições de Longa Permanência. Revista Neurocienciências, São Paulo, v. 23, n. 3, p. 397-404, 2015.

RICHARDSON, Roberto et al. Pesquisa social: métodos e técnicas. 3. ed. São Paulo: Atlas, 2012

SANTOS, Flávia; ANDRADE, Vivian; BUENO, Orlando. Envelhecimento: um processo multifatorial. Psicologia em Estudo, Maringá, v. 14, n. 1, p. 3-10, 2009. 
SANTOS, Roberto; VIRTUOSO JÚNIOR, Jair. Confiabilidade da versão brasileira da escala de Atividades Instrumentais da Vida Diária. Revista Brasileira de Promoção da Saúde, Fortaleza, v. 21, n. 4, p. 290-296, 2008.

SOBRAL, Ana; ARAUJO, Cláudia; SOBRAL, Marcos. Mild cognitive impairment in the elderly Relationship between communication and functional capacity. Dementia $e$ Neuropsychologia, São Paulo, v. 12, n. 2, p. 165-172, 2018.

TRINDADE, Ana et al. Repercussão do declínio cognitivo na capacidade funcional em idosos institucionalizados e não institucionalizados. Fisioterapia em Movimento, Curitiba, v. 26, n. 2, p. 281-289, 2013.

Data de Submissão: 10/09/2018

Data de Aprovação: 10/10/2018 